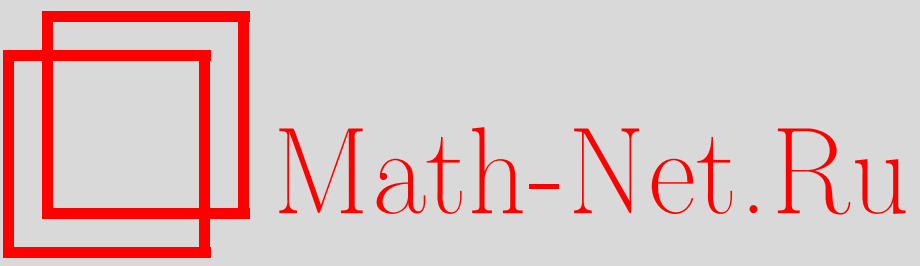

В. В. Корнев, А. П. Хромов, Классическое решение смешанной задачи для однородного волнового уравнения с закрепленными концами, Итоги науки и техн. Сер. Соврем. мат. и ее прил. Темат. обз., 2019, том 172, 119-133

DOI: https://doi.org/10.36535/0233-6723-2019-172-119-133

Использование Общероссийского математического портала Math-Net.Ru подразумевает, что вы прочитали и согласны с пользовательским соглашением

http: //www.mathnet.ru/rus/agreement

Параметры загрузки:

IP: 3.89 .197 .203

26 апреля 2023 г., $16: 27: 23$ 


\title{
КЛАССИЧЕСКОЕ РЕШЕНИЕ СМЕШАННОЙ ЗАДАЧИ ДЛЯ ОДНОРОДНОГО ВОЛНОВОГО УРАВНЕНИЯ С ЗАКРЕПЛЕННЫМИ КОНЦАМИ
}

\author{
(c) 2019 г. $\quad$ B. В. KOPHEB, А. П. ХРОМОВ
}

\begin{abstract}
АннотАция. При помощи метода Фурье найдены необходимые и достаточные условия существования классического решения смешанной задачи для однородного волнового уравнения с суммируемым потенциалом и закрепленными концами и получено явное представление решения в виде быстро сходящегося ряда.
\end{abstract}

Ключевые слова: смешанная задача, волновое уравнение, суммируемый потенциал, метод Фурье.

\section{CLASSICAL SOLUTION OF THE MIXED PROBLEM FOR A HOMOGENEOUS WAVE EQUATION WITH FIXED ENDPOINTS}

\author{
(c) 2019 V. V. KORNEV, A. P. KHROMOV
}

\begin{abstract}
Using the Fourier method, we obtain necessary and sufficient conditions for the existence of a classical solution of the mixed problem for a homogeneous wave equation with summable potential and fixed endpoints and also obtain an explicit representation of the solution in the form of a rapidly converging series.
\end{abstract}

Keywords and phrases: mixed task, wave equation, summable potential, Fourier method.

AMS Subject Classification: 34B45, 35L05

В статье будет рассмотрена следующая задача:

$$
\begin{gathered}
\frac{\partial^{2} u(x, t)}{\partial t^{2}}=\frac{\partial^{2} u(x, t)}{\partial x^{2}}-q(x) u(x, t), \quad x \in[0,1], \quad t \geqslant 0, \\
u(0, t)=u(1, t)=0, \\
u(x, 0)=\varphi(x), \quad u_{t}^{\prime}(x, 0)=0 .
\end{gathered}
$$

Считаем, что $q(x), \varphi(x)$ - комплекснозначные функции, причем $q(x) \in L[0,1]$. В [1] получены условия на $\varphi(x)$, достаточные для существования классического решения по методу Фурье задачи (1)-(3) (уравнение (1) удовлетворяется почти всюду). Эти условия таковы: $\varphi(x), \varphi^{\prime}(x)$ абсолютно непрерывны, $\varphi(0)=\varphi(1)=0$ и

$$
L \varphi=-\varphi^{\prime \prime}(x)+q(x) \varphi(x) \in L_{p}[0,1], \quad p>1 .
$$

Эти условия, кроме условия (4), являются необходимыми для классического решения. В этой статье мы убираем условие (4). Тем самым получаем необходимые и достаточные условия для 
существования классического решения задачи (1)-(3). Настоящая работа является развитием направления, содержащегося в [2-4].

1. Единственность классического решения. В этом разделе рассмотрим вопрос о единственности решения для более общего случая. Именно, займемся следующей задачей:

$$
\begin{gathered}
\frac{\partial^{2} u(x, t)}{\partial t^{2}}=\frac{\partial^{2} u(x, t)}{\partial x^{2}}-q(x) u(x, t)+f(x, t), \quad x \in[0,1], \quad t \geqslant 0, \\
u(0, t)=u(1, t)=0, \\
u(x, 0)=\varphi(x), \quad u_{t}^{\prime}(x, 0)=\psi(x),
\end{gathered}
$$

где все функции, входящие в (5)-(7), комплекснозначные, причем $q(x) \in L[0,1], f(x, t) \in L\left(Q_{T}\right)$ при любых $T>0$; здесь $Q_{T}=[0,1] \times[0, T]$.

Под классическим решением понимаем функцию $u(x, t)$, непрерывную вместе с $u_{x}^{\prime}(x, t)$ и $u_{t}^{\prime}(x, t)$, причем $u_{x}^{\prime}(x, t)$ и $u_{t}^{\prime}(x, t)$ абсолютно непрерывны по $x$ (по $\left.t\right)$, удовлетворяющую условиям (6), (7) и почти всюду по $x$ и $t$ уравнению (5). Поэтому в случае классического решения необходимо считать, что $\varphi(x), \varphi^{\prime}(x)$ и $\psi(x)$ абсолютно непрерывны, $\varphi(0)=\varphi(1)=\psi(0)=\psi(1)=0$, $\varphi^{\prime \prime}(x) \in L[0,1], \psi^{\prime}(x) \in L[0,1]$.

Теорема 1. Если и(x,t) классическое решение задачи (5)-(7), причем дополнительно $\partial^{2} u(x, t) / \partial t^{2} \in L\left[Q_{T}\right]$ при любом $T>0$, то оно единственно и находится по формуле

$$
u(x, t)=-\frac{1}{2 \pi i}\left(\int_{\lambda \mid=r}+\sum_{n \geqslant n_{0}} \int_{\gamma_{n}}\right)\left[\left(R_{\lambda} \varphi\right) \cos \rho t+\left(R_{\lambda} \psi\right) \frac{\sin \rho t}{\rho}+\int_{0}^{t} R_{\lambda}(f(\cdot, \tau)) \frac{\sin \rho(t-\tau)}{\rho} d \tau\right] d \lambda,
$$

в которой ряд справа при любом фиксированном $t \geqslant 0$ сходится абсолютно и равномерно по $x \in[0,1]$.

Здесь $R_{\lambda}=(L-\lambda E)^{-1}$ - резольвента оператора $L: L y=-y^{\prime \prime}(x)+q(x) y(x), y(0)=y(1)=0(\lambda-$ спектральный параметр, $E$ - единичный оператор). $R_{\lambda}(f(\cdot, \tau))$ означает, что $R_{\lambda}$ применяется к $f(x, \tau)$ по $x, \lambda=\rho^{2}, \Re \rho \geqslant 0, \gamma_{n}$-образ в $\lambda$-плоскости окружности $\tilde{\gamma}_{n}=\{\rho|| \rho-n \pi \mid=\delta\}$, $\delta>0$ достаточно мало, $r$ достаточно велико и фиксировано, $n_{0}$-такой номер, что при $n \geqslant n_{0}$ внутри $\gamma_{n}$ находится по одному собственному значению оператора $L$ и все $\gamma_{n}$ при $n \geqslant n_{0}$ находятся вне окружности $|\lambda|=r$.

Доказательство теоремы 1. При фиксированном $t u(x, t)$ как функция $x$ принадлежит области определения оператора $L$. Поэтому по теореме о разложении по собственным и присоединенным функциям оператора $L$ имеем

$$
u(x, t)=-\frac{1}{2 \pi i}\left(\int_{\lambda \mid=r}+\sum_{n \geqslant n_{0}} \int_{\gamma_{n}}\right) R_{\lambda}(u(\cdot, t)) d \lambda,
$$

и ряд (9) сходится абсолютно и равномерно по $x \in[0,1]$. Положим $R_{\lambda} u(\cdot, t)=y(x, t, \lambda)$. Тогда

$$
L y=L R_{\lambda} u=(L-\lambda E+\lambda E) R_{\lambda} u=u(x, t)+\lambda R_{\lambda} u=u(x, t)+\lambda y(x, t, \lambda) .
$$

Привлекая функцию Грина (ядро интегрального оператора $R_{\lambda}$ ), находим

$$
y(x, t, \lambda)=\int_{0}^{1} G(x, \xi, \lambda) u(\xi, t) d \xi \quad \Longrightarrow \quad y_{t}^{\prime}(x, t, \lambda)=\int_{0}^{1} G(x, \xi, \lambda) u_{t}^{\prime}(\xi, t) d \xi .
$$

Поскольку $u(x, t)$ есть классическое решение задачи (5)-(7), то

$$
R_{\lambda} u_{t t}^{\prime \prime}=-R_{\lambda}(L u)+R_{\lambda} f(\cdot, t) .
$$


Из (10) получаем

$$
y_{t}^{\prime}(x, t, \lambda)=\int_{0}^{1} G(x, \xi, \lambda) u_{t}^{\prime}(\xi, 0) d \xi+\int_{0}^{1} G(x, \xi, \lambda) d \xi \int_{0}^{t} u_{t t}^{\prime \prime}(\xi, \tau) d \tau .
$$

Но $G(x, \xi, \lambda) u_{t t}^{\prime \prime}(\xi, \tau) \in L\left[Q_{T}\right]$ по переменным $\xi$ и $\tau$; поэтому согласно теореме Фубини имеем

$$
\int_{0}^{1} G(x, \xi, \lambda) u_{t t}^{\prime \prime}(\xi, \tau) d \xi \in L[0, T]
$$

по $\tau$. Значит,

$$
y_{t}^{\prime}(x, t, \lambda)=\int_{0}^{1} G(x, \xi, \lambda) u_{t}^{\prime}(\xi, 0) d \xi+\int_{0}^{t} d \tau \int_{0}^{1} G(x, \xi, \lambda) u_{t t}^{\prime \prime}(\xi, \tau) d \xi .
$$

Отсюда следует, что $y_{t}^{\prime}(x, t, \lambda)$ абсолютно непрерывна по $t$ и

$$
y_{t t}^{\prime \prime}(x, t, \lambda)=\int_{0}^{1} G(x, \xi, \lambda) u_{t t}^{\prime \prime}(\xi, t) d \xi
$$

почти при всех $t$. Из этой формулы в силу (11) имеем

$$
y_{t t}^{\prime \prime}(x, t, \lambda)=R_{\lambda} u_{t t}^{\prime \prime}=R_{\lambda}(-L u+f) .
$$

Но $R_{\lambda}(L-\lambda E+\lambda E) u=u(x, t)+\lambda R_{\lambda} u=u(x, t)+\lambda y(x, t, \lambda)$; следовательно,

$$
y_{t t}^{\prime \prime}(x, t, \lambda)=-u(x, t)-\lambda y(x, t, \lambda)+R_{\lambda} f(\cdot, t),
$$

или

$$
y_{t t}^{\prime \prime}(x, t, \lambda)+\lambda y(x, t, \lambda)=-u(x, t)+R_{\lambda} f(\cdot, t)
$$

Кроме того,

$$
y(x, 0, \lambda)=R_{\lambda} \varphi, \quad y_{t}^{\prime}(x, 0)=R_{\lambda} \psi .
$$

Из общего решения уравнение (12) при начальных условиях из (13) получаем

$$
y(x, t, \lambda)=\left(R_{\lambda} \varphi\right) \cos \rho t+\left(R_{\lambda} \psi\right) \frac{\sin \rho t}{\rho}+\int_{0}^{t} \frac{\sin \rho(t-\tau)}{\rho}\left[-u(x, \tau)+R_{\lambda} f(\cdot, \tau)\right] d \tau .
$$

Теперь, в силу того факта, что

$$
\int_{0}^{t} \frac{\sin \rho(t-\tau)}{\rho} u(x, \tau) d \tau
$$

является целой функцией по $\lambda$, из (9) получаем (8).

Формула (8) является формулой формального решения задачи (5)-(7) по методу Фурье (схожие с (8) формулы имеются в [5]- [6]).

2. Преобразование формального решения по методу Фурье. Рассмотрим сначала следующую задачу:

$$
\begin{gathered}
\frac{\partial^{2} u(x, t)}{\partial t^{2}}=\frac{\partial^{2} u(x, t)}{\partial x^{2}}+f(x, t), \quad x \in[0,1], \quad t \geqslant 0, \\
u(0, t)=u(1, t)=0 \\
u(x, 0)=u_{t}^{\prime}(x, 0)=0 .
\end{gathered}
$$


Теорема 2. Если $f(x, t) \in L\left[Q_{T}\right]$, то ряд (8) формального решения задачи (14)-(16) по методу Фурье сходится при всех $x$ и $t$, и его сумма есть

$$
u(x, t)=\frac{1}{2} \int_{0}^{t} d \tau \int_{x-t+\tau}^{x+t-\tau} \tilde{f}(\eta, \tau) d \eta
$$

где функиия $\tilde{f}(\eta, \tau)$ нечетна и 2-периодична по $\eta$, причем $\tilde{f}(\eta, \tau)=f(\eta, \tau)$ при $\eta \in[0,1]$.

Эта теорема доказана в [7] для $f(x, t) \in L_{2}\left[Q_{T}\right]$ и в [8] для $f(x, t) \in L\left[Q_{T}\right]$ при любом $T>0$.

Замечание 1. Правая часть (17) имеет смысл при всех $x, t \in(-\infty,+\infty) \times[0, \infty]$.

Пусть $u(x, t)$ - классическое решение задачи (1)-(3) с условием $\partial^{2} u(x, t) / \partial t^{2} \in L\left[Q_{T}\right]$ при любом $T>0$. Тогда $\varphi(x), \varphi^{\prime}(x)$ абсолютно непрерывны и $\varphi(0)=\varphi(1)=0$. По теореме 1 имеет место формула

$$
u(x, t)=-\frac{1}{2 \pi i}\left(\int_{|\lambda|=r}+\sum_{n \geqslant n_{0}} \int_{\gamma_{n}}\right)\left(R_{\lambda} \varphi\right) \cos \rho t d \lambda,
$$

и ряд (18) при каждом $t \geqslant 0$ сходится абсолютно и равномерно по $x \in[0,1]$. Представим ряд (18) в виде

$$
u(x, t)=u_{0}(x, t)+u_{1}(x, t),
$$

где $u_{0}(x, t)$ - ряд (18), в котором $R_{\lambda}$ заменено на $R_{\lambda}^{\circ}, u_{1}(x, t)$ - ряд (18), в котором $R_{\lambda}$ заменено на $R_{\lambda}-R_{\lambda}^{\circ}, R_{\lambda}^{\circ}=\left(L_{0}-\lambda E\right)^{-1}$ и $L_{0}$ равно $L$ при $q(x)=0$.

Лемма 1. Сумма ряда $u_{0}(x, t)$ равна $a_{0}(x, t)$, где $a_{0}(x, t)=\frac{1}{2}[\tilde{\varphi}(x+t)+\tilde{\varphi}(x-t)]$, где $\tilde{\varphi}(x)$ нечетна и 2-периодична по $x \in(-\infty,+\infty)$ и $\tilde{\varphi}(x)=\varphi(x)$ при $x \in[0,1]$. Ряд $u_{1}(x, t)$ сходится при каждом $t$ абсолютно и равномерно по $x \in[0,1]$.

Доказательство. По теореме вычетов ряд $u_{0}(x, t)$ есть

$$
\begin{aligned}
& \text { где }(f, g)=\int_{0}^{1} f(x) g(x) d x \text {. Отсюда следует, что } \\
& \quad u_{0}(x, t)=\sum_{+}+\sum_{-}, \quad \text { где } \quad \sum_{ \pm}=\sum_{n=1}^{\infty}(\varphi(\xi), \sin n \pi \xi) \sin n \pi(x \pm t) .
\end{aligned}
$$

$$
u_{0}(x, t)=2 \sum_{n=1}^{\infty}(\varphi(\xi), \sin n \pi \xi) \sin n \pi x \cos n \pi t,
$$

Поскольку $u(x, t)$ - классическое решение, в силу необходимых условий на $\varphi(x)$ ряд

$$
\sum_{n=1}^{\infty}(\varphi(\xi), \sin n \pi \xi) \sin n \pi x
$$

сходится абсолютно и равномерно по $x$ на всей оси, и его сумма есть $\frac{1}{2} \tilde{\varphi}(x)$. Значит,

$$
u_{0}(x, t)=\frac{1}{2}[\tilde{\varphi}(x+t)+\tilde{\varphi}(x-t)] .
$$

Наконец, из теоремы 1 и полученной сходимости ряда $u_{0}(x, t)$ следует утверждение леммы для ряда $u_{1}(x, t)$. Лемма доказана.

Лемма 2. Функиия $a_{0}(x, t)$ непрерывна и непрерывно дифференцируема по $x, t \in(-\infty,+\infty) \times$ $[0, \infty]$, функиия $a_{0 x}^{\prime}(x, t)\left(a_{0 t}^{\prime}(x, t)\right)$ абсолютно непрерывна по $x$ (соответственно, по $t$ ),

$$
\frac{\partial^{2} a_{0}(x, t)}{\partial t^{2}}=\frac{\partial^{2} a_{0}(x, t)}{\partial x^{2}}
$$

почти всюду по $x$ и по $t$ и выполняются условия

$$
\begin{gathered}
a_{0}(0, t)=a_{0}(1, t)=0, \\
a_{0}(x, 0)=\tilde{\varphi}(x), \quad a_{0 t}^{\prime}(x, 0)=0 .
\end{gathered}
$$


Доказательство. Непрерывность $a_{0}(x, t)$ по $x$ и $t$ следует из доказательства леммы 1 . Рассмотрим $\tilde{\varphi}^{\prime}(x)$. Очевидно, что $\tilde{\varphi}^{\prime}(x)$ непрерывна всюду, кроме, быть может, $x=n$ при целом $n$. Далее, из равенства $\tilde{\varphi}^{\prime}(-x)=\tilde{\varphi}^{\prime}(x)$ следует $\tilde{\varphi}^{\prime}(-0)=\tilde{\varphi}^{\prime}(+0)$, т.е. $\tilde{\varphi}^{\prime}(x)$ непрерывна в нуле и, следовательно, во всех точках $x=n$ при четном $n$. Наконец, из равенств

$$
\tilde{\varphi}^{\prime}\left(-(1+0)=\tilde{\varphi}^{\prime}(-1-0)=\tilde{\varphi}^{\prime}(1-0)=\tilde{\varphi}^{\prime}(-1+0)=\tilde{\varphi}^{\prime}(1+0)\right)
$$

следует, что $\tilde{\varphi}^{\prime}(x)$ непрерывна в точке $x=1$ и, следовательно, во всех точках $x=n$ при нечетном $n$. Значит, $\tilde{\varphi}^{\prime}(x) \in C(-\infty, \infty)$; так как эта функция абсолютно непрерывна при $x \in[0,1]$, то $\tilde{\varphi}^{\prime}(x)$ абсолютно непрерывна при всех $x \in(-\infty,+\infty)$. Значит, почти при всех $x$ и $t$ выполняется (20). Проверим выполнение (21)-(22). Имеем

$$
a_{0}(1, t)=\frac{1}{2}[\tilde{\varphi}(1+t)+\tilde{\varphi}(1-t)]=\frac{1}{2}[\tilde{\varphi}(1+t)+\tilde{\varphi}(-1-t)]=0,
$$

т.е. верно (21). Проверим (22). Очевидно, имеем $a_{0}(x, 0)=\tilde{\varphi}(x)$. Из соотношения

$$
a_{0 t}^{\prime}(x, t)=\frac{1}{2}\left[\tilde{\varphi}^{\prime}(x+t)-\tilde{\varphi}^{\prime}(x-t)\right]
$$

получаем $a_{0 t}^{\prime}(x, 0)=0$. Лемма доказана.

Следствие. При $x \in[0,1]$ функция $a_{0}(x, t)$ является классическим решением задачи $(1)-(3)$ при $q(x)=0$ с условием единственности

$$
\frac{\partial^{2} a_{0}(x, t)}{\partial t^{2}} \in L\left[Q_{T}\right]
$$

Приступаем к исследованию ряда $u_{1}(x, t)$. В силу леммы 2 его сумма (для которой сохраняем обозначение $\left.u_{1}(x, t)\right)$ является классическим решением задачи

$$
\begin{gathered}
\frac{\partial^{2} u_{1}(x, t)}{\partial t^{2}}=\frac{\partial^{2} u_{1}(x, t)}{\partial x^{2}}-q(x) u_{1}(x, t)+f_{0}(x, t) \\
u_{1}(0, t)=u_{1}(1, t)=0 \\
u_{1}(x, 0)=u_{1 t}^{\prime}(x, 0)=0
\end{gathered}
$$

где $f_{0}(x, t)=-q(x) a_{0}(x, t)$, с условием единственности. Поэтому согласно теореме 1 функция $u_{1}(x, t)$ является суммой ряда своего формального решения, которое по формуле (8) есть

$$
u_{1}(x, t)=-\frac{1}{2 \pi i}\left(\int_{\lambda \mid=r}+\sum_{n \geqslant n_{0}} \int_{\gamma_{n}} \int_{0}^{t} R_{\lambda}\left(f_{0}(\cdot, \tau)\right) \frac{\sin \rho(t-\tau)}{\rho} d \tau d \lambda .\right.
$$

По аналогии с (19) представим (26) в виде

$$
u_{1}(x, t)=u_{10}(x, t)+u_{2}(x, t) .
$$

Здесь $u_{10}(x, t)$ - функция $(26)$, где вместо $R_{\lambda}$ берется $R_{\lambda}^{\circ}$. Так как $u_{10}(x, t)-$ сумма формального решения уравнения

$$
\frac{\partial^{2} u_{10}(x, t)}{\partial t^{2}}=\frac{\partial^{2} u_{10}(x, t)}{\partial x^{2}}+f_{0}(x, t)
$$

с условиями (24)-(25), где вместо $u_{1}(x, t)$ берем $u_{10}(x, t)$, то по теореме 2

$$
u_{10}(x, t)=\frac{1}{2} \int_{0}^{t} d \tau \int_{x-t+\tau}^{x+t-\tau} \tilde{f}_{0}(\eta, \tau) d \eta
$$

Обозначим через $\tilde{q}(x)$ четное 2-периодическое продолжение функции $q(x)$ с промежутка $x \in$ $[0,1]$ на всю ось. Тогда очевидно, что $\tilde{f}_{0}(\eta, \tau)=-\tilde{q}(\eta) a_{0}(\eta, \tau)$. 
Обозначим через П множество всех нечетных и 2-периодических по $x$ функций $f(x, t)$ на всей оси $(t-$ параметр). Таким образом, П является образом линейной операции $\tilde{f}(x, t)$ продолжения $f(x, t)$ по $x$ с отрезка $[0,1]$ на всю ось. Легко видеть, что если $f(x, t) \in \Pi$, то и

$$
\int_{0}^{t} d \tau \int_{x-t+\tau}^{x+t-\tau} f(\eta, \tau) d \eta \in \Pi
$$

Очевидно, также, что если $f(x, t) \in \Pi$, то и $\tilde{q}(x) f(x, t) \in \Pi$. В дальнейшем будем опускать знак у $\tilde{q}(x)$, т.е. теперь $q(x)$ задана на оси, четна и 2 -периодична. Таким образом, имеем $\tilde{f}_{0}(\eta, \tau)=$ $-q(\eta) a_{0}(\eta, \tau)$.

Так как правая часть (27) определена при всех $x, t \in(-\infty,+\infty) \times[0, \infty]$, то ее будем обозначать теперь $a_{1}(x, t)$, т.е. $a_{1}(x, t)$ - нечетное 2 -периодическое продолжение $u_{10}(x, t)$ по $x$ на всю ось $x$.

Займемся теперь $a_{1}(x, t)$. Непрерывность $a_{1}(x, t)$ по $x$ и $t$ следует из $(27)$ и того факта, что $\tilde{f}_{0}(x, t)$ суммируема по $x$ и $t$ в любой ограниченной области (в дальнейшем $\tilde{f}_{0}(x, t)$ будет обозначать через $f_{0}(x, t)$; это же будет относиться и к другим подобным функциям). Далее, из-за нечетности и 2-периодичности $f_{0}(x, t)$ по $x$ имеем

$$
\int_{-t+\tau}^{t-\tau} f_{0}(\eta, \tau) d \eta=\int_{1-t+\tau}^{1+(t-\tau)} f_{0}(\eta, \tau) d \eta=\int_{-(t-\tau)}^{t-\tau} f_{0}(1+\xi, \tau) d \xi=0 .
$$

Поэтому $a_{1}(0, t)=a_{1}(1, t)=0$. Очевидно еще, что $a_{1}(x, 0)=0$.

Лемма 3. Функиия $a_{1}(x, t)$ непрерывно дифферениируема по $x$ и $t$, причем

$$
\begin{aligned}
& \frac{\partial a_{1}(x, t)}{\partial t}=J_{11}(x, t)+J_{21}(x, t), \\
& \frac{\partial a_{1}(x, t)}{\partial x}=J_{11}(x, t)-J_{21}(x, t),
\end{aligned}
$$

где

$$
J_{11}(x, t)=\frac{1}{2} \int_{x}^{x+t} f_{0}(\xi, x+t-\xi) d \xi, \quad J_{21}(x, t)=\frac{1}{2} \int_{x-t}^{x} f_{0}(\xi, \xi-x+t) d \xi
$$

u $J_{11}(x, t), J_{21}(x, t)$ непрерывны по $x$ u $t$.

Доказательство. Докажем, что $a_{1}(x, t)$ непрерывно дифференцируема по $t$. Сначала предположим, что $q(\eta)$ непрерывна. Тогда очевидно, что $a_{1}(x, t)$ непрерывно дифференцируема по $t$ и

$$
\frac{\partial a_{1}(x, t)}{\partial t}=\frac{1}{2} \int_{0}^{t}\left[f_{0}(x+t-\tau, \tau)+f_{0}(x-t+\tau, \tau)\right] d \tau=J_{11}(x, t)+J_{21}(x, t) .
$$

Пусть теперь $q(x) \in L[0,1]$. Тогда очевидно, что $J_{11}(x, t)$ и $J_{21}(x, t)$ непрерывны по $x$ и $t$. Используя теорему Фубини, имеем

$$
\begin{gathered}
2 \int_{0}^{t}\left[J_{11}(x, \tau)+J_{21}(x, \tau)\right] d \tau=\int_{0}^{t} d \tau \int_{\tau}^{0} f_{0}(x+\tau-\eta, \eta)(-d \eta)+\int_{0}^{t} d \tau \int_{0}^{\tau} f_{0}(\eta+x-\tau, \eta) d \eta= \\
=\int_{0}^{t} d \eta \int_{\eta}^{t} f_{0}(x+\tau-\eta, \eta) d \tau+\int_{0}^{t} d \eta \int_{\eta}^{t} f_{0}(\eta+x-\tau, \eta) d \tau= \\
=\int_{0}^{t} d \eta \int_{x}^{x+t-\eta} f_{0}(\xi, \eta) d \xi+\int_{0}^{t} d \eta \int_{x}^{x+\eta-t} f_{0}(\xi, \eta)(-d \xi)=\int_{0}^{t} d \eta \int_{x-t+\eta}^{x+t-\eta} f_{0}(\xi, \eta) d \xi=2 a_{1}(x, t) .
\end{gathered}
$$


Значит, $a_{1}(x, t)$ непрерывно дифференцируема по $t$ и выполняется $(28)$.

Если $f_{0}(x, t)$ непрерывна, то очевидно, что $a_{1}(x, t)$ непрерывно дифференцируема по $x$ и справедлива формула (29).

Пусть теперь $q(x) \in L[0,1]$. Положим

$$
Q(x, t)=J_{11}(x, t)-J_{21}(x, t) .
$$

Тогда

$$
\begin{aligned}
2 \int_{0}^{x} Q(\xi, t) d \xi & =\int_{0}^{t} d \tau \int_{0}^{x}\left[f_{0}(\xi+t-\tau, \tau)+f_{0}(\xi-t+\tau, \tau)\right] d \xi= \\
& =\int_{0}^{t} d \tau\left[\int_{t-\tau}^{x+t-\tau} f_{0}(\eta, \tau)-\int_{-t+\tau}^{x-t+\tau} f_{0}(\eta, \tau)\right] d \eta+\int_{0}^{t} d \tau \int_{-t+\tau}^{t-\tau} f_{0}(\eta, \tau) d \eta= \\
& =\int_{0}^{t} d \tau \int_{x-t+\tau}^{x+t-\tau} f_{0}(\eta, \tau) d \eta=2 a_{1}(x, t) .
\end{aligned}
$$

Отсюда следует непрерывная дифференцируемость $a_{1}(x, t)$ по $x$ и формула $(29)$.

Лемма 4. Если функиия $a(x)$ суммируема, а $b(x)$ непрерывна, то функиия

$$
\Phi(x)=\int_{0}^{x} a(\eta) b(\eta) d \eta
$$

абсолютно непрерывна, $\Phi^{\prime}(x)$ существует и конечна во всякой точке $x$, в которой существуют и конечны

$$
\frac{d}{d x} \int_{0}^{x} a(\eta) d \eta, \quad \frac{d}{d x} \int_{0}^{x}|a(\eta)| d \eta
$$

Эта лемма очевидна.

Лемма 5. При фиксированном $x$ функция $J_{11}(x, t)$ абсолютно непрерывна по $t u$

$$
2 \frac{\partial J_{11}(x, t)}{\partial t}=-\left(q(x+t) a_{0}(x+t, 0)+\int_{x}^{x+t} q(\xi) a_{0 t}^{\prime}(\xi, x+t-\xi) d \xi\right)
$$

почти при всех $t \in[0, \infty)$, причем правая часть (30) конечна, если конечны величины

$$
\frac{d}{d \xi} \int_{0}^{\xi} q(\tau) d \tau, \quad \frac{d}{d \xi} \int_{0}^{\xi}|q(\tau)| d \tau
$$

при $\xi=x+t$.

Доказательство. Сначала предположим, что функция $q(x)$ непрерывна на оси. Тогда функция $J_{11}(x, t)$ непрерывно дифференцируема по $t$ и при всех $t$ верно соотношение (30).

Пусть теперь $q(x) \in L[0,1]$. Тогда правая часть (30) имеет смысл, причем интеграл в $(30)$ есть непрерывная функция по $x$ и $t$. Обозначим правую часть $(30)$ через $-G(t)$. Тогда $G(t)$ суммируема. Далее, имеем

$$
\int_{0}^{t} G(\eta) d \eta=\int_{0}^{t} f_{0}(x+\eta, 0) d \eta+\int_{0}^{t} d \eta \int_{x}^{x+\eta} f_{0 t}^{\prime}(\xi, x+\eta-\xi) d \xi
$$


где $f_{0 t}^{\prime}$ означает дифференцирование $f_{0}$ по второй переменной. Пользуясь теоремой Фубини, переставим порядки интегрирования в двойном интеграле $J$ в $(32)$. Обозначив через $\varepsilon(x, t)$ функцию Хевисайда $(\varepsilon(x, t)=1$ при $x \geqslant t, \varepsilon(x, t)=0$ при $x<t)$, получим

$$
\begin{aligned}
J=\int_{0}^{t} d \eta \int_{x}^{x+t} \varepsilon(x+\eta, \xi) f_{0 t}^{\prime}(\xi, x+\eta-\xi) d \xi=\int_{x}^{x+t} d \xi \int_{0}^{t} \varepsilon(x+\eta, \xi) f_{0 t}^{\prime}(\xi, x+\eta-\xi) d \eta= \\
=\int_{x}^{x+t} d \xi \int_{\xi-x}^{t} f_{0 t}^{\prime}(\xi, x+\eta-\xi) d \eta=-\int_{x}^{x+t} q(\xi) d \xi \int_{\xi-x}^{t} a_{0 t}^{\prime}(\xi, x+t-\xi) d \eta= \\
=-\int_{x}^{x+t} q(\xi)\left[a_{0}(\xi, x+t-\xi)-a_{0}(\xi, 0)\right] d \xi
\end{aligned}
$$

( $a_{0 t}^{\prime}$ имеет тот же смысл, что и $f_{0 t}^{\prime}$ выше). Поэтому

$$
\int_{0}^{t} G(\eta) d \eta=\int_{x}^{x+t} f_{0}(\xi, x+t-\xi) d \xi=2 J_{11}(x, t)
$$

Значит, $J_{11}(x, t)$ абсолютно непрерывна по $t$ и имеет место (30), причем правая часть (30) конечна по лемме 4 , если конечны величины (31) при $\xi=x+t$. Лемма доказана.

Лемма 6. При фиксированном $t$ функиия $J_{11}(x, t)$ абсолютно непрерывна по $x$ u

$$
2 \frac{\partial J_{11}(x, t)}{\partial x}=-\left[q(x+t) a_{0}(x+t, 0)-q(x) a_{0}(x, t)+\int_{x}^{x+t} q(\xi) a_{0 t}^{\prime}(\xi, x+t-\xi) d \xi\right]
$$

почти всюду по $x$, где правая часть конечна, если конечны величины (31) nри $\xi=x, x+t$.

Доказательство. Сначала предположим, что $q(x) \in C(-\infty, \infty)$. Тогда функция $J_{11}(x, t)$ непрерывно дифференцируема по $x$ и имеет место соотношение (33).

Пусть теперь $q(x) \in L[0,1]$. Тогда правая часть (33) имеет смысл, причем интеграл в (33) есть непрерывная функция по $(x, t) \in(-\infty,+\infty) \times[0, \infty)$. Обозначим правую часть (33) через $G(x)$; эта функция суммируема по $x$. Далее, имеем

$$
\int_{0}^{x} G(\xi) d \xi=\int_{0}^{x} f_{0}(\xi+t, 0) d \xi-\int_{0}^{x} f_{0}(\xi, t) d \xi+\int_{0}^{x} d \xi \int_{\xi}^{\xi+t} f_{0 t}^{\prime}(\eta, \xi+t-\eta) d \eta .
$$

Пользуясь теоремой Фубини, переставим порядки интегрирования в двойном интеграле $J$ в (34):

$$
J=\int_{0}^{x} d \xi \int_{0}^{x+t} \varepsilon(\xi+t, \eta) \varepsilon(\eta, \xi) f_{0 t}^{\prime}(\eta, \xi+t-\eta) d \eta=\int_{0}^{x+t} d \eta \int_{0}^{x} \varepsilon(\xi+t, \eta) \varepsilon(\eta, \xi) f_{0 t}^{\prime}(\eta, \xi+t-\eta) d \xi .
$$

Во внутреннем интеграле $J_{1}$ в (35) сделаем замену $\xi+t-\eta=\tau$. Тогда

$$
\begin{aligned}
J_{1}=\int_{t-\eta}^{x+t-\eta} \varepsilon(\eta+\tau, \eta) \varepsilon(\eta, \tau-t+\eta) f_{0 t}^{\prime}(\eta, \tau) d \tau= & \\
& =\int_{\max \{0, t-\eta\}}^{\min \{t, x+t-\eta\}} f_{0 t}^{\prime}(\eta, \tau) d \tau=f_{0}(\eta, \min \{t, x+t-\eta\})-f_{0}(\eta, \max \{0, t-\eta\}) .
\end{aligned}
$$


Значит,

$$
J=\int_{0}^{x+t} f_{0}(\eta, \min \{t, x+t-\eta\}) d \eta-\int_{0}^{x+t} f_{0}(\eta, \max \{0, t-\eta\}) d \eta .
$$

Рассмотрим первый интеграл $J_{2}$ в $(36)$. Пусть $t \leqslant x$. Тогда

$$
J_{2}=\int_{0}^{t}+\int_{t}^{x}+\int_{x}^{x+t}
$$

Имеем

$$
\int_{0}^{t}=\int_{0}^{t} f_{0}(\eta, t) d \eta, \quad \int_{t}^{x}=\int_{t}^{x} f_{0}(\eta, t) d \eta, \quad \int_{x}^{x+t}=\int_{x}^{x+t} f_{0}(\eta, x+t-\eta) d \eta .
$$

Значит, при $t \leqslant x$

$$
J_{2}=\int_{0}^{x} f_{0}(\eta, t) d \eta+\int_{x}^{x+t} f_{0}(\eta, x+t-\eta) d \eta .
$$

Пусть теперь $t \geqslant x$. Тогда

$$
\begin{aligned}
J_{2}=\int_{0}^{x}+\int_{x}^{t}+\int_{t}^{x+t}=\int_{0}^{x} f_{0}(\eta, t) d \eta & +\int_{x}^{t} f_{0}(\eta, x+t-\eta) d \eta+ \\
& +\int_{t}^{x+t} f_{0}(\eta, x+t-\eta) d \eta=\int_{0}^{x} f_{0}(\eta, t) d \eta+\int_{x}^{x+t} f_{0}(\eta, x+t-\eta) d \eta .
\end{aligned}
$$

Таким образом, при $t \leqslant x$ и при $t \geqslant x$ имеет место одна и та же формула:

$$
J_{2}=\int_{0}^{x} f_{0}(\eta, t) d \eta+\int_{x}^{x+t} f_{0}(\eta, x+t-\eta) d \eta .
$$

Рассмотрим теперь второй интеграл $J_{3}$ в $(36)$. Пусть $t \leqslant x$. Тогда имеем

$$
\begin{aligned}
J_{3}=\int_{0}^{t}+\int_{t}^{x}+\int_{x}^{x+t}=\int_{0}^{t} f_{0}(\eta, t-\eta) d \eta+\int_{t}^{x} f_{0}(\eta, 0) d \eta+ & \int_{x}^{t} f_{0}^{t+t} f_{0}(\eta, 0) d \eta \\
& =\int_{0}^{x+t} f_{0}(\eta, t-\eta) d \eta+\int_{t}^{x} f_{0}(\eta, 0) d \eta .
\end{aligned}
$$

Пусть теперь $t \geqslant x$. Тогда

$$
\begin{aligned}
J_{3}=\int_{0}^{x}+\int_{x}^{t}+\int_{t}^{x+t}=\int_{0}^{x} f_{0}(\eta, t-\eta) d \eta+\int_{x}^{t} f_{0}(\eta, t-\eta) d \eta+ & \\
& +\int_{t}^{x+t} f_{0}(\eta, 0) d \eta=\int_{0}^{t} f_{0}(\eta, t-\eta) d \eta+\int_{t}^{x+t} f_{0}(\eta, 0) d \eta .
\end{aligned}
$$

Значит, и для $J_{3}$ справедлива одна и та же формула как при $t \leqslant x$, так и при $t \geqslant x$.

Таким образом,

$$
J=\int_{0}^{x} f_{0}(\eta, t) d \eta+\int_{x}^{x+t} f_{0}(\eta, t-\eta) d \eta-\int_{0}^{t} f_{0}(\eta, t-\eta) d \eta-\int_{t}^{x+t} f_{0}(\eta, 0) d \eta .
$$


Итак,

$$
\begin{aligned}
\int_{0}^{x} G(\xi) d \xi=\int_{0}^{x} f_{0}(\xi+t, 0) d \xi-\int_{0}^{x} f_{0}(\xi, t) d \xi & +\int_{0}^{x} f_{0}(\xi, t) d \xi+ \\
& +\int_{x}^{t+t} f_{0}(\xi, x+t-\xi) d \xi-\int_{0}^{t} f_{0}(\xi, t-\xi) d \xi-\int_{t}^{x+t} f_{0}(\xi, 0) d \xi= \\
& =\int_{x}^{x+t} f_{0}(\xi, x+t-\xi) d \xi-\int_{0}^{t} f_{0}(\eta, t-\eta) d \eta .
\end{aligned}
$$

Таким образом,

$$
G(x)=f_{0}(x+t, 0)-f_{0}(x, t)+\int_{x}^{x+t} f_{0 t}^{\prime}(\xi, x+t-\xi) d \xi
$$

почти всюду по $x$, т.е. $G(x)$ конечна, если конечны величины $(31)$ при $\xi=x$ и $\xi=x+t$. Лемма доказана.

Аналогично леммам 5 и 6 получаются следующие леммы 7 и 8.

Лемма 7. При фиксированном $x$ функция $J_{21}(x, t)$ абсолютно непрерывна по $t u$

$$
\frac{\partial J_{21}(x, t)}{\partial t}=-\left[q(x-t) a_{0}(x-t, 0)+\int_{x-t}^{x} q(\xi) a_{0 t}^{\prime}(\xi, \xi-x+t) d \xi\right]
$$

почти при всех $t$, причем правая часть конечна, если при $\xi=x+t$ конечны величины (31).

Лемма 8. При фиксированном $t$ функция $J_{21}(x, t)$ абсолютно непрерывна по $x$ u

$$
\frac{\partial J_{21}(x, t)}{\partial x}=-\left[q(x) a_{0}(x, t)-q(x-t) a_{0}(x-t, 0)-\int_{x-t}^{x} q(\xi) a_{0 t}^{\prime}(\xi, \xi-x+t) d \xi\right]
$$

почти при всех $x \in(-\infty, \infty)$, где правая часть конечна, если при $\xi=x, x-t$ конечны величиныс (31).

С помощью лемм 3-8 получаем следующий результат.

Теорема 3. Если и (x,t)-классическое решение задачи (1)-(3) с условием единственности, то $a_{1}(x, t)$ непрерывна и непрерывно дифференцируема по $(x, t) \in(-\infty,+\infty) \times[0, \infty)$, функиия $a_{1 x}^{\prime}(x, t)\left(a_{1 t}^{\prime}(x, t)\right)$ абсолютно непрерывна по $x$ (соответственно, по $\left.t\right)$, для $a_{1}(x, t)$ выполняются условия (24)-(25) и

$$
\frac{\partial^{2} a_{1}(x, t)}{\partial t^{2}}=\frac{\partial^{2} a_{1}(x, t)}{\partial x^{2}}-q(x) a_{0}(x, t)
$$

почти всюду по $(x, t) \in(-\infty,+\infty) \times[0, \infty)$, где $q(x)$ - четная 2-периодическая функиия, $q(x) \in$ $L[0,1]$, причем равенство (37) имеет место при всех $x$ u $t$, где величины (31) конечны при $\xi=x, x-t, x+t$. Здесъ $\partial^{2} a_{1}(x, t) / \partial t^{2} \in L\left[Q_{T}\right]$.

Теперь будем исследовать $u_{1}(x, t)$ по той же схеме, что $u(x, t)$ выше, т.е., исходим из того, что $u_{1}(x, t)$ - классическое решение задачи (23)-(25) с условием $\partial^{2} u_{1}(x, t) / \partial t^{2} \in L\left[Q_{T}\right]$; в итоге получим

$$
u_{1}(x, t)=a_{2}(x, t)+u_{2}(x, t),
$$

где для $a_{2}(x, t)$ имеет место та же формула, что и выше для $a_{1}(x, t)$, где $\tilde{f}_{0}(\eta, \tau)$ заменяется на $f_{1}(\eta, \tau)=-q(\eta) a_{1}(\eta, \tau)$, а $u_{2}(x, t)$ - классическое решение задачи, полученной из $(23)-(25)$ 
заменой $u_{1}(x, t)$ на $u_{2}(x, t)$ и $f_{0}(x, t)$ на $f_{1}(x, t)$. Этот процесс может быть бесконечно продолжен, т.е. получаем

$$
u_{n}(x, t)=a_{n}(x, t)+u_{n+1}(x, t),
$$

где $a_{n}(x, t)$ - то же, что и $a_{1}(x, t)$, с заменой $f_{0}(\eta, \tau)$ на $f_{n-1}(\eta, \tau)\left(f_{k}(\eta, \tau)=-q(\eta) a_{k}(\eta, \tau)\right)$ и $u_{n+1}(x, t)$ - решение системы, получаемой из $(23)-(25)$ заменой $u_{1}(x, t)$ на $u_{n+1}(x, t)$ и $f_{0}(x, t)$ на $f_{n}(x, t)=-q(x) a_{n}(x, t)$.

Для $a_{n}(x, t)$ по индукции получаем следующую теорему, аналогичную теореме 3.

Теорема 4. Если и $(x, t)$ - классическое решение задачи (1)-(3) с условием единственности, то функиия $a_{n}(x, t)$ при $n \geqslant 2$ непрерывна и непрерывно дифференцируема по $(x, t) \in(-\infty,+\infty) \times$ $[0, \infty)$, функиця $a_{n, x}^{\prime}(x, t)\left(a_{n, t}^{\prime}(x, t)\right)$ абсолютно непрерывна по $x$ (соответственно, по $\left.t\right)$, для $a_{n}(x, t)$ выполняются условия $(24)-(25) u$

$$
\frac{\partial^{2} a_{n}(x, t)}{\partial t^{2}}=\frac{\partial^{2} a_{n}(x, t)}{\partial x^{2}}-q(x) a_{n-1}(x, t)
$$

почти всюду по $(x, t) \in(-\infty,+\infty) \times[0, \infty)$, где функиия $q(x)$ четна, 2-периодична и $q(x) \in L[0,1]$, причем равенство (38) имеет место при всех $x$ и $t$, где величины (31) конечны при $\xi=x$, $x-t, x+t$.

Лемма 9. Имеют место формуль

$$
u(x, t)=A_{n}(x, t)+\Omega_{n}(x, t), \quad n=2, \ldots,
$$

где

$$
\begin{gathered}
A_{n}(x, t)=\sum_{k=0}^{n} a_{k}(x, t), \\
\Omega_{n}(x, t)=-\frac{1}{2 \pi i}\left(\int_{\lambda \mid=r}+\sum_{n \geqslant n_{0}} \int_{\gamma_{n}}\right)\left[\int_{0}^{t}\left(R_{\lambda}-R_{\lambda}^{\circ}\right)\left(f_{n-1}(\cdot, \tau)\right) \frac{\sin \rho(t-\tau)}{\rho} d \tau\right] d \lambda .
\end{gathered}
$$

Доказательство. Имеем

$$
u(x, t)=A_{n-1}(x, t)+u_{n}(x, t) .
$$

Далее, $u_{n}(x, t)$ есть классическое решение задачи, полученной из $(23)-(25)$ заменой $u_{1}(x, t)$ на $u_{n}(x, t)$ и $f_{0}(x, t)$ на $f_{n-1}(x, t)$ с условием единственности. Поэтому по теореме 1

$$
u_{n}(x, t)=-\frac{1}{2 \pi i}\left(\int_{\lambda \mid=r}+\sum_{n \geqslant n_{0}} \int_{\gamma_{n}}\right)\left[\int_{0}^{t} R_{\lambda}(f(\cdot, \tau)) \frac{\sin \rho(t-\tau)}{\rho} d \tau\right] d \lambda=a_{n+1}(x, t)+\Omega_{n}(x, t) .
$$

Лемма 10. Пусть $T$ - произвольное положительное число, $m$-наименьшее натуральное число, для которого $T \leqslant m$. Тогда

$$
\left\|a_{n}(x, t)\right\|_{C\left[Q_{T}\right]} \leqslant M_{1}\left(\frac{M_{2}}{2}\right)^{n-1} \frac{T^{n-1}}{(n-1) !}, \quad n=1,2, \ldots,
$$

где $M_{1}=\left\|a_{n}(x, t)\right\|_{C\left[Q_{T}\right]}, M_{2}=(2 m+1)\|q\|_{1}$ (здесь $\|\cdot\|_{1}-$ норма в $\left.L[0,1]\right)$. Кроме того, $M_{1} \leqslant$ $C_{T}\|\varphi\|_{1}$ и постоянная $C_{T}$ не зависит от $\varphi(x)$.

Доказательство. Очевидно $f_{n}(x, t) \in L\left[Q_{T}\right], a_{n}(x, t) \in C\left[Q_{T}\right], n=1,2, \ldots$ При $n=1$ оценка

$$
\left|a_{n}(x, t)\right| \leqslant M_{1}\left(\frac{M_{2}}{2}\right)^{n-1} \frac{t^{n-1}}{(n-1) !}
$$


очевидно выполняется. Предположим, что она выполняется для некоторого $n$, и докажем, что она выполняется для $n+1$. Имеем

$$
\begin{aligned}
& \left|a_{n+1}\right| \leqslant \frac{1}{2} \int_{0}^{t} d \tau \int_{x-t+\tau}^{x+t-\tau}\left|f_{n}(\eta, \tau)\right| d \eta \leqslant \\
& \quad \leqslant \frac{1}{2} \int_{0}^{t} d \tau \int_{-m}^{m+1}\left|f_{n}(\eta, \tau)\right| d \eta \leqslant \frac{2 m+1}{2} \int_{0}^{t} d \tau \int_{0}^{1}|q(\eta)|\left|a_{n}(\eta, \tau)\right| d \eta \leqslant \\
& \leqslant \frac{2 m+1}{2} \int_{0}^{t} d \tau \int_{0}^{1}|q(\eta)| M_{1}\left(\frac{M_{2}}{2}\right)^{n-1} \frac{\tau^{n-1}}{(n-1) !} d \eta=M_{1}\left(\frac{M_{2}}{2}\right)^{n} \frac{t^{n}}{n !} .
\end{aligned}
$$

Следовательно, (39) верно и для $n+1$. Оценим $M_{1}$. Имеем

$$
\begin{gathered}
\left|a_{1}(x, t)\right| \leqslant \frac{1}{2} \int_{0}^{t} d \tau \int_{x-t+\tau}^{x+t-\tau}\left|f_{0}(\eta, \tau)\right| d \eta \leqslant \frac{1}{2} \int_{0}^{T} d \tau \int_{-m}^{m+1}\left|f_{0}(\eta, \tau)\right| d \eta= \\
=\frac{2 m+1}{2} \int_{0}^{T} d \tau \int_{0}^{1}\left|f_{0}(\eta, \tau)\right| d \eta \leqslant \frac{2 m+1}{2} \int_{0}^{T} d \tau \int_{0}^{1}|q(\eta)|(|\tilde{\varphi}(\eta+\tau)|+|\tilde{\varphi}(\eta-\tau)|) d \eta \leqslant \\
\leqslant \frac{2 m+1}{2} \int_{0}^{1}|q(\eta)| d \eta 2 m \int_{0}^{1}|\varphi(\tau)| d \tau=C_{T}\|\varphi\|_{1} .
\end{gathered}
$$

Теорема 5. Если $и(x, t)$ - классическое решение задачи (1)-(3) с условием $\partial^{2} u(x, t) \partial t^{2} \in$ $L\left[Q_{T}\right], m o$

$$
u(x, t)=A(x, t)=\sum_{n=0}^{\infty} a_{n}(x, t),
$$

причем ряд сходится абсолютно и равномерно по $(x, t) \in Q_{T}$ при любом $T>0$.

Доказательство. По лемме 10 ряд $A(x, t)$ сходится абсолютно и равномерно по $(x, t) \in Q_{T}$ при любом $T>0$. Далее, при $\rho \in \tilde{\gamma}_{n}$ имеем оценки:

$$
\left|\left(R_{\lambda}-R_{\lambda}^{\circ}\right)\left(f_{n}(\cdot, \tau)\right) \frac{\sin \rho(t-\tau)}{\rho}\right| \leqslant \frac{C}{n^{3}} \int_{0}^{1}\left|f_{n}(x, \tau)\right| d \tau,
$$

где $C$ не зависит от $\eta, \tau$, и по лемме 10

$$
\int_{0}^{1}\left|f_{n}(x, \tau)\right| d \tau \leqslant M_{1}\left(\frac{M_{2} T}{2}\right)^{n-1} \frac{1}{(n-1) !}\|q\|_{1} .
$$

Замечание 2. Ряд $A(x, t)$ сходится абсолютно и равномерно в любой ограниченной области по $(x, t) \in(-\infty,+\infty) \times[0, \infty)$.

Таким образом, ряд $A(x, t)$ является преобразованием ряда формального решения, полученное путем многократного использования рекомендаций А. Н. Крылова по ускорению сходимости рядов.

При $q(x)=0$ формула (40) переходит в формулу Даламбера. Поэтому формула (40) представляет собой аналог формулы Даламбера и является формулой явного решения смешанной задачи. 
Замечание 3. Формулы, схожие с $(40)$ в случае $q(x) \in C^{1}[0,1]$ и других граничных условий, были получены ранее Б. М. Левитаном без привлечения метода Фурье (см. [9]).

3. Классическое решение. Ряд $A(x, t)$ можно составить, взяв в качестве $\varphi(x)$ любую суммируемую функцию. В этом разделе считаем, что в этом ряде функция $\varphi(x)$ такова, что $\varphi(x), \varphi^{\prime}(x)$ абсолютно непрерывны и $\varphi(0)=\varphi(1)=0$. При этих условиях на $\varphi(x)$ мы установим, что сумма ряда $A(x, t)$ есть классическое решение задачи (1)-(3).

Лемма 11. Сумма $A(x, t)$ ряда (40) непреръьна и непрерывно дифферениируема по $(x, t) \in$ $(-\infty,+\infty) \times[0, \infty)$, причем

$$
\begin{aligned}
& \frac{\partial A(x, t)}{\partial t}=\frac{\partial a_{0}(x, t)}{\partial t}-\frac{1}{2} \int_{x}^{x+t} q(\xi) A(\xi, x+t-\xi) d \xi-\frac{1}{2} \int_{x-t}^{x} q(\xi) A(\xi, \xi-x+t) d \xi \\
& \frac{\partial A(x, t)}{\partial x}=\frac{\partial a_{0}(x, t)}{\partial x}-\frac{1}{2} \int_{x}^{x+t} q(\xi) A(\xi, x+t-\xi) d \xi+\frac{1}{2} \int_{x-t}^{x} q(\xi) A(\xi, \xi-x+t) d \xi .
\end{aligned}
$$

Доказательство. По лемме 10 ряд $A(x, t)$ сходится абсолютно и равномерно в любой ограниченной области переменных $x$ и $t$. Значит, сумма ряда $A(x, t)$ непрерывна по $x$ и $t$. Далее, формально дифференцируем ряд $A(x, t)$ почленно по $t$ :

$$
\frac{\partial A(x, t)}{\partial t}=\frac{\partial a_{0}(x, t)}{\partial t}+\sum_{1}^{\infty} \frac{\partial a_{n}(x, t)}{\partial t} .
$$

Из рассуждений п. 2 следует, что построение и исследование функции $a_{n}(x, t)$ не связано со смешанной задачей, а лишь с формулой $(32)$, где $f_{0}(\eta, t)$ заменено на $-\frac{1}{2} q(\eta) a_{n-1}(\eta, \tau)$. Поэтому по индукции имеем

$$
\frac{\partial a_{n}(x, t)}{\partial t}=J_{1 n}(x, t)+J_{2 n}(x, t)
$$

где

$$
\begin{aligned}
& J_{1 n}(x, t)=-\frac{1}{2} \int_{x}^{x+t} q(\xi) a_{n-1}(\xi, x+t-\xi) d \xi \\
& J_{2 n}(x, t)=-\frac{1}{2} \int_{x-t}^{x} q(\xi) a_{n-1}(\xi, \xi-x+t) d \xi .
\end{aligned}
$$

По лемме 10 в любой ограниченной области изменения $x$ и $t$ имеем оценки:

$$
\left|J_{j n}(x, t)\right| \leqslant C \frac{C_{1}^{n-1}}{(n-1) !}
$$

где $C$ и $C_{1}$ зависят лишь от ограниченной области изменения $x$ и $t$. Отсюда следует, что в этой области ряд $\sum_{1}^{\infty} \frac{\partial a_{n}(x, t)}{\partial t}$ сходится абсолютно и равномерно, и потому функция $A(x, t)$ непрерывно дифференцируема по $t$ и имеет место формула (43). Далее, по теореме Лебега о предельном переходе имеем

$$
\begin{aligned}
\sum_{1}^{\infty} \int_{x}^{x+t} q(\xi) a_{n-1}(\xi, x+t-\xi) d \xi & =\lim _{N \rightarrow \infty} \sum_{1}^{N} \int_{x}^{x+t} q(\xi) a_{n-1}(\xi, x+t-\xi) d \xi= \\
=\lim _{N \rightarrow \infty} \int_{x}^{x+t} q(\xi) \sum_{1}^{N} a_{n-1}(\xi, x+t-\xi) d \xi & =\int_{x}^{x+t} q(\xi) A(\xi, x+t-\xi) d \xi
\end{aligned}
$$


Аналогично

$$
\sum_{1}^{\infty} \int_{x-t}^{x} q(\xi) a_{n-1}(\xi, \xi-x+t) d \xi=\int_{x-t}^{x} q(\xi) A(\xi, \xi-x+t) d \xi
$$

и тем самым получаем (41). Аналогично получается (42). Лемма доказана.

Теорема 6. Если $\varphi(x), \varphi^{\prime}(x)$ абсолютно непрерывны и $\varphi(0)=\varphi(1)=0$, то сумма рлда $A(x, t)$ является классическим решением задачи (1)-(3) с условием единственности.

Доказательство. Введем обозначения

$$
B_{1}(x, t)=\int_{x}^{x+t} q(\xi) A(\xi, x+t-\xi) d \xi, \quad B_{2}(x, t)=\int_{x-t}^{x} q(\xi) A(\xi, \xi-x+t) d \xi .
$$

По аналогии с леммой 5 согласно лемме 11 получаем, что функция $B_{1}(x, t)$ при фиксированном $x$ абсолютно непрерывна по $t$,

$$
\frac{\partial B_{1}(x, t)}{\partial t}=q(x+t) A(x+t, 0)+\int_{x}^{x+t} q(\xi) A_{t}^{\prime}(\xi, x+t-\xi) d \xi
$$

почти всюду по $t$ и правая часть конечна, если при $\xi=x+t$ конечны величины (31).

По аналогии с леммой 7 при фиксированном $x$ функция $B_{2}(x, t)$ абсолютно непрерывна по $t$ и

$$
\frac{\partial B_{2}(x, t)}{\partial t}=q(x-t) A(x-t, 0)+\int_{x-t}^{x} q(\xi) A_{t}^{\prime}(\xi, \xi-x+t) d \xi
$$

при всех $t$, для которых при $\xi=x-t$ конечны величины (31).

По аналогии с леммой 6 функция $B_{1}(x, t)$ при фиксированном $t$ абсолютно непрерывна по $x$ и

$$
\frac{\partial B_{1}(x, t)}{\partial x}=q(x+t) A(x+t, 0)-q(x) A(x, t)+\int_{x}^{x+t} q(\xi) A_{t}^{\prime}(\xi, x+t-\xi) d \xi
$$

если при $\xi=x, x+t$ конечны величины (31).

По аналогии с леммой 8 заключаем, что функция $B_{2}(x, t)$ при фиксированном $t$ абсолютно непрерывна по $x$ и

$$
\frac{\partial B_{2}(x, t)}{\partial x}=q(x) A(x, t)-q(x-t) A(x-t, 0)-\int_{x-t}^{x} q(\xi) A_{t}^{\prime}(\xi, \xi-x+t) d \xi
$$

если при $\xi=x, x-t$ конечны величины (31).

Так как

$$
\begin{aligned}
& \frac{\partial A(x, t)}{\partial t}=\frac{\partial a_{0}(x, t)}{\partial t}-\frac{1}{2} B_{1}(x, t)-\frac{1}{2} B_{2}(x, t), \\
& \frac{\partial A(x, t)}{\partial x}=\frac{\partial a_{0}(x, t)}{\partial x}-\frac{1}{2} B_{1}(x, t)+\frac{1}{2} B_{2}(x, t),
\end{aligned}
$$

то $\partial A(x, t) / \partial t$ при фиксированном $x$ абсолютно непрерывна по $t$ и

$$
\frac{\partial^{2} A(x, t)}{\partial t^{2}}=\frac{\partial^{2} a_{0}(x, t)}{\partial t^{2}}-\frac{1}{2} \frac{\partial B_{1}(x, t)}{\partial t}-\frac{1}{2} \frac{\partial B_{2}(x, t)}{\partial t}
$$

почти всюду по $t$, причем (44) имеет место, если при $\xi=x+t, x-t$ конечны величины (31), $\tilde{\varphi}^{\prime \prime}(x+t)$ и $\tilde{\varphi}^{\prime \prime}(x-t)$.

Наконец, $\partial A(x, t) / \partial t$ при фиксированном $t$ абсолютно непрерывна по $x$ и

$$
\frac{\partial^{2} A(x, t)}{\partial x^{2}}=\frac{\partial^{2} a_{0}(x, t)}{\partial x^{2}}-\frac{1}{2} \frac{\partial B_{1}(x, t)}{\partial x}-\frac{1}{2} \frac{\partial B_{2}(x, t)}{\partial x} .
$$


Так как очевидно, что $\partial^{2} a_{0}(x, t) / \partial t^{2} \in L[0,1]$, то получаем решение в классе единственности. Теорема доказана.

Замечание 4. Как и в доказательстве теоремы 3, получаем, что соотношения (1)-(3) выполняется при всех $x$ и $t$, только $A(x, 0)=\varphi(x)$ заменяется на $A(x, 0)=\tilde{\varphi}(x)$ и в уравнении $(1)$ функция $q(x)$ четна и 2-периодична на всей оси.

\section{СПИСОК ЛИТЕРАТУРЫ}

1. Хромов А. П. О сходимости формального решения по методу Фурье волнового уравнения с суммируемым потенциалом// Ж. вычисл. мат. мат. физ. - 2016. - 56, № 10. - С. 1795-1809.

2. Корнев В. В., Хромов А. П. Об обобщенном формальном решении по методу Фурье смешанной задачи для неодородного волнового уравнения// 19 Междунар. Саратов. зимняя школа «Современные проблемы теории функций и их приближений», посв. 90-летию акад. П. Л. Ульянова (Саратов, 29 января - 2 февраля 2018). - Саратов: СГУ. - С. 156-159.

3. Корнев В. В., Хромов А. П. О решении неоднородного волнового уравнения с закрепленными концами и нулевыми начальными условиями// 19 Междунар. Саратов. зимняя школа «Современные проблемы теории функций и их приближений», посв. 90-летию акад. П. Л. Ульянова (Саратов, 29 января 2 февраля 2018). - Саратов: СГУ. - С. 159-160.

4. Корнев В. В., Хромов А. П. О классическом и обобщенном решении смешанной задачи для волнового уравнения// в кн.: Современные методы теории краевых задач/ Междунар. конф. «Понтрягинские чтения-XXIX», посв. 90-летию акад. В. А. Ильина (Москва, 2-6 мая). - М., 2018. - С. 132-133.

5. Расулов М. Л. Метод контурного интеграла. - М.: Наука, 1964.

6. Вагабов А. И. Введение в спектральную теорию дифференциальных операторов. - Ростов: РГУ, 1994.

7. Корнев В. В., Хромов А. П. Смешанная задача для неоднородного волнового уравнения с суммируемым потенциалом // Ж. вычисл. мат. мат. физ. -2017 . -57 , № 10. - С. 1692-1707.

8. Корнев В. В., Хромов А. П. Сходимость формального решения по методу Фурье в смешанной задаче для простейшего неоднородного волнового уравнения// в кн.: Математика. Механика (сб. науч. тр.). - Саратов: СГУ, 2017. - 19. - С. 41-44.

9. Левитан Б. М. Операторы обобщенного сдвига и некоторые их применения. - М.: Физмалит, 1965.

Корнев Владимир Викторович

Саратовский национальный исследовательский государственный университет

им. Н. Г. Чернышевского

E-mail: KornevVV@info.sgu.ru

Хромов Август Петрович

Саратовский национальный исследовательский государственный университет

им. Н. Г. Чернышевского

E-mail: khromovap@info.sgu.ru 